\title{
The current status of an FDG-PET cancer screening program in Japan, based on a 4-year (2006-2009) nationwide survey
}

\author{
Ryogo Minamimoto $\cdot$ Michio Senda $\cdot$ Seishi Jinnouchi $\cdot$ Takashi Terauchi $\cdot$ Tsuyoshi Yoshida $\cdot$ \\ Takeshi Murano $\cdot$ Hiroshi Fukuda $\cdot$ Takeshi Iinuma $\cdot$ Kimiichi Uno $\cdot$ Sadahiko Nishizawa \\ Eriko Tsukamoto $\cdot$ Hiroshi Iwata $\cdot$ Takeshi Inoue $\cdot$ Kazuhiro Oguchi $\cdot$ Rumi Nakashima $\cdot$ Tomio Inoue
}

Received: 29 June 2012/ Accepted: 17 September 2012/Published online: 20 October 2012

(C) The Japanese Society of Nuclear Medicine 2012

\begin{abstract}
Objective The aim of this study was to survey the 18F-fluorodeoxyglucose-positron emission tomography (FDG-PET) cancer screening program conducted in Japan. Methods The "FDG-PET cancer screening program" included both FDG-PET and positron emission tomography with computed tomography (PET/CT) with or without other combined screening tests that were performed for
\end{abstract}

\section{R. Minamimoto $(\square)$}

Division of Nuclear Medicine, Department of Radiology,

National Center for Global Health and Medicine,

1-21-1 Toyama, Shinjyuku-ku, Tokyo 162-8655, Japan

e-mail: ryogominamimoto@yahoo.co.jp

\section{R. Minamimoto $\cdot$ T. Inoue}

Department of Radiology, Yokohama City University,

Graduate School of Medicine, Yokohama, Japan

\section{Senda}

Division of Molecular Imaging, Institute of Biomedical Research and Innovation, Kobe, Japan

\section{S. Jinnouchi}

Atsuchi Memorial Institute of Radiology, Atsuchi Memorial

Clinic PET Center, Kagoshima, Japan

\section{T. Terauchi $\cdot$ T. Murano}

Screening Technology and Development Division,

Research Center for Cancer Prevention and Screening,

National Cancer Center, Tokyo, Japan

\section{T. Yoshida}

Department of Radiology, Koga Hospital 21 PET Center,

Kurume, Japan

H. Fukuda

Department of Nuclear Medicine and Radiology,

Institute of Development, Aging and Cancer,

Tohoku University, Sendai, Japan cancer screening in asymptomatic subjects. A total of 155,456 subjects who underwent the FDG-PET cancer screening program during 2006-2009 were analyzed.

Results Of the 155,456 subjects, positive findings suggesting possible cancer were noted in 16,955 (10.9\%). The number of cases with detected cancer was $1,912(1.23 \%$ of the total screened cases, annual range 1.14-1.30\%). Of the 1,912 cases of detected cancer, positive findings on FDGPET were present in 1,491 cases $(0.96 \%$ of the total

\section{T. Iinuma}

National Institute of Radiological Sciences, Chiba, Japan

K. Uno

Gaien-higashi Clinic, Tokyo, Japan

\section{S. Nishizawa}

Hamamatsu Medical Imaging Center,

Hamamatsu Medical Photonics Foundation,

Hamamatsu, Japan

E. Tsukamoto

Central CI Clinic, Sapporo, Japan

H. Iwata

Department of Radiology, Nagoya PET Imaging Center,

Nagoya, Japan

T. Inoue

Department of Radiology, Ehime Prefectural Central Hospital,

Matsuyama, Japan

K. Oguchi

Positron Imaging Center, Aizawa Hospital, Matsumoto, Japan

R. Nakashima Japanese Red Cross Kumamoto Health Care Center, Kumamoto, Japan 
number of screened cases). According to the results of further examinations, the true positive rate for subjects with suggested possible cancer (positive predictive value) was $32.3 \%$ with FDG-PET. Cancers of the colon/rectum, thyroid, lung, and breast were most frequently found (396, 353, 319, and 163 cases, respectively) with high PET sensitivity $(85.9,90.7,86.8,84.0 \%$, respectively). Prostate cancer and gastric cancer (165 and 124 cases, respectively) had low PET sensitivity (37.0 and $37.9 \%$, respectively). The Union for International Cancer Control (UICC) clinical stage of cancer found with the FDG-PET cancer screening program was mainly Stage I.

Conclusions The FDG-PET screening program in Japan has detected a variety of cancers at an early stage. However, several cancers were found in repeated FDG-PET cancer screening program, indicating the limitation of a one-time FDG-PET cancer screening program. The value of the FDG-PET cancer screening program is left to the judgment of individuals with regard to its potentials and limitations.

\section{Keywords FDG $\cdot$ PET $\cdot$ PET/CT $\cdot$ Cancer screening}

\section{Introduction}

Screening is the investigation of a group of asymptomatic individuals in order to detect a disease that has a high probability of development. The National Cancer Institute (NCI) estimates that appropriate cancer screening can prevent $3-35 \%$ of premature deaths caused by cancer. The NCI suggests that cancer screening might decrease cancer morbidity because treatment for earlier-stage cancers is often less aggressive than that for more advanced cancers. Only a few screening methods have evidence supporting their use in reducing cancer-related mortality [1].

Fluorine-18 fluorodeoxyglucose (FDG)-positron emission tomography (PET) and positron emission tomography/ computed tomography (PET/CT) have had a considerable impact in the field of oncology in terms of differentiating between benign and malignant tumors, staging cancers, evaluating the effectiveness of treatment, and predicting prognosis. PET and PET/CT can provide whole-body imaging, and have the potential to reveal malignancies anywhere in the body. The performance of FDG-PET for cancer screening was first published by the HIMEDIC Imaging Center at Lake Yamanaka. They established a cancer screening program which consisted of an FDG-PET examination combined with screening tests such as ultrasonography and computed tomography to compensate for the limitations of FDG-PET [2]. Since then, cancer screening using FDG-PET based on Yamanaka's method has become widespread in Japan, and is performed in more than 130 facilities. The performance profile of FDG-PET cancer screening in 2005 was reported. A total of 43,996 healthy subjects underwent FDG-PET (including PET/CT) scanning with or without other tests for cancer screening in 38 PET centers in Japan [3]. The yearly survey was continued until 2009 in Japan. PET cancer screening is more widespread than before, and PET/CT scanners were more widely used than ever. The potential of the FDG-PET cancer screening program is investigated in the present report through the results of 155,456 cases that underwent FDG-PET cancer screening from 2006 to 2009. This report aims at clarifying the performance of FDG-PET cancer screening, but does not attempt to confirm nor deny the effectiveness of the PET cancer screening program as a cancer screening method.

\section{Materials and methods}

\section{Subjects}

All study protocols in this retrospective observation study were approved by our institutional review board. Questionnaires regarding the FDG-PET cancer screening program were obtained from subjects who had undergone FDG-PET and/or other combined screening tests, and were referred for further evaluation. Subjects underwent FDG-PET cancer screening in a total of 233 facilities (2006, 59; 2007, 57; 2008, 58; and 2009, 59). The total number of subjects was 43,061 (25,594 men and 17,467 women) in 2006, 39,867 (23,948 men and 15,919 women) in 2007, 38,929 (23,055 men, 15,230 women, and 644 gender unidentified) in 2008, and 33,599 (20,142 men and 13,457 women) in 2009 for a total of $155,456(92,739$ men, 62,073 women, and 644 unidentified) from a total of 233 facilities. Results based on these 155,456 cases are discussed in the present report. The facilities that participated in the questionnaire survey are listed in Table 1.

\section{Contents of the investigation}

The questionnaire regarding the FDG-PET cancer screening consists of an "Investigation of facilities" section that describes the situation of each facility, and an "Investigation of suspected cancer cases" section that describes the inspection of suspected cancer cases. The "Investigation of facilities" section surveyed the following areas: (1) the type and methods of FDG-PET (FDG-PET machines, injected radioactive of FDG, presence and method of attenuation correction, time to obtain PET or PET/CT, screening range, performance of delayed scanning and start time); (2) characteristics of the CT scanner integrated in the PET/CT system (mAs, CT image reconstruction interval); (3) fixed or 
Table 1 Cooperated PET centers for the survey

\begin{tabular}{|c|c|c|}
\hline Asahikawa-Kosei General Hospital & University of Fukui Hospital & Tsukazaki Clinic \\
\hline Keiyukai Sapporo Hospital & $\begin{array}{l}\text { Sugita Genpaku Memorial Obama Municipal } \\
\text { Hospital }\end{array}$ & $\begin{array}{l}\text { Institute of Biomedical Research and } \\
\text { Innovation }\end{array}$ \\
\hline Sapporo Minamisanjo Hospital & Fukui Red Cross Hospital & Himeji Central Hospital's Clinic \\
\hline Central CI Clinic & Fukui-ken Saiseikai Hospital & Agape Kabutoyama Hospital \\
\hline LSI Sapporo Clinic & Iida Municipal Hospital & Sumoto Itsuki Hospital \\
\hline Tomakomai City Hospital & Aizawa Hospital & Kouseikai Takai Hospital \\
\hline Aomori PET Diagnostic Imaging Center & Ichinose Neurosurgical Hospital & Nishinokyo Hospital \\
\hline Akita Research Institute of Brain and Blood Vessels & Kizawame Memorial Hospital & Wakayama-minami Radiology Clinic \\
\hline Sendai Kousei Hospital & Juntendo University Shizuoka Hospital & Tottori University Hospital \\
\hline Kousei Sendai Clinic & Seirei Hamamatsu General Hospital & Tottori Municipal Hospital \\
\hline Southern Tohoku General Hospital, Miyagi & Hamamatsu Medical Imaging Center & Kawasaki Medical School Hospital \\
\hline Southern Tohoku General Hospital, Fukushima & Nagoya Central Hospital & Kurashiki Central Hospital \\
\hline Shirakawa Kosei General Hospital & Daiyukai Daiichi Hospital & Tsuyama Central Hospital \\
\hline Dokkyo Medical University Hospital & KARIYA TOYOTA General Hospital & Okayama Diagnostic Imaging Center \\
\hline Utsunomiya Central Clinic & Nagoya PET Imaging Center & Okayama Kyokuto Hospital \\
\hline Kusunoki Hospital & East Nagoya Imaging Diagnosis Center & Okamura Isshindow Hospital \\
\hline Saitama Medical University & Koseikai Hospital & Chuden Hospital \\
\hline Tokorozawa PET Diagnostic Imaging Clinic & $\begin{array}{l}\text { Handa Medical Association Health Care } \\
\text { Center }\end{array}$ & Hiroshima Heiwa Clinic \\
\hline Asahi General Hospital & Mie University Hospital & St. Hill Hospital \\
\hline Sannou Medical Center & Iga City Medical Checkup Center & Tokushima University Hospital \\
\hline Yotsukaido Tokushukai Hospital & Saiseikai Matsusaka General Hospital & Kagawa University Hospital \\
\hline $\begin{array}{l}\text { Nippon Medical School Clinical Imaging Center for } \\
\text { Healthcare }\end{array}$ & Mie Advanced Medical PET Center & Takinomiya General Hospital \\
\hline National Center for Global Health and Medicine & Kusatsu General Hospital & Shikoku Cancer Center \\
\hline Toranomon Hospital & Oumikusatsu Tokusyukai Hospital & Ehime Prefectural Central Hospital \\
\hline $\begin{array}{l}\text { Japanese Foundation for Cancer Research Cancer } \\
\text { Screening Center }\end{array}$ & Nishijin Hospital & Kochi University Hospital \\
\hline Nishidai Clinic & Mitsubishi Kyoto Hospital & Kyushu University Hospital \\
\hline Yotsuya Medical Cube & Takeda Oncologic Positron Imaging Center & $\begin{array}{l}\text { Kurume University School of } \\
\text { Medicine }\end{array}$ \\
\hline Musashimurayama Hospital & $\begin{array}{l}\text { Kinki University Institute of Advanced } \\
\text { Clinical Medicine }\end{array}$ & Kouhoukai Takagi Hospital \\
\hline Iwai Medical Foundation Medicheck Imaging Center & Saiseikai Nakatsu Hospital, Osaka & Koga Hospital 21 \\
\hline Yuai Clinic & Panasonic Health Care Center & $\begin{array}{l}\text { Fukuoka Wajiro PET Diagnostic } \\
\text { Imaging Clinic }\end{array}$ \\
\hline Shonan-Atsugi Clinic & Hanwa Intelligent Medical Center & Kitakyushu PET Center \\
\hline General Sagami Kosei Hospital & Higashitemma Clinic & Nishi Isahaya Hospital \\
\hline Toyama PET Imaging Center & OCAT Clinic & Saiseikai Kumamoto Hospital \\
\hline Public Central Hospital of Matto Ishikawa & HIMEDIC Clinic West & Oita Diagnostic Imaging Center \\
\hline Asanogawa General Hospital & MI Clinic & $\begin{array}{l}\text { Kagoshima-kyousaikai Nanpuh } \\
\text { Hospital }\end{array}$ \\
\hline $\begin{array}{l}\text { Tousenkai Healthcare System Keiju PET-CT, Linac } \\
\text { Center }\end{array}$ & Morinomiya Clinic & Atsuchi Memorial Clinic \\
\hline Kanazawa Cardiovascular Hospital & Ishikiriseiki Hospital & \\
\hline
\end{tabular}

optional other cancer screening tests combined with FDGPET; (4) total number, gender, and age of subjects who underwent FDG-PET cancer screening; (5) number of repeated annual FDG-PET cancer screening (at least 2 consecutive years); (6) number, gender, age of subjects with positive findings by FDG-PET and/or combined screening test suggesting possible cancer; (7) number of positive/ negative findings by FDG-PET and/or combined screening 
tests in the case of suggested possible cancer; (8) number of excluded cancer cases with combined screening tests after cancer was suspected by FDG-PET.

Detailed information was required only for possible cancer cases in the "Investigation of suspected cancer cases" section. Five types of investigation sheets were provided: "lung cancer," "colon/rectum cancer," "thyroid cancer," and "breast cancer" (which are all frequently detected by FDG-PET), and "other cancers". The "Investigation of suspected cancer cases" section asked about the following: (1) gender; (2) age; (3) past history of cancer; (4) with or without of repeated annual FDG-PET cancer screening and other suspected cancers by previous FDG-PET screening if any; (5) findings of FDG-PET and delayed imaging if present; (6) results of the combined cancer screening tests; and (7) final results of further detailed examinations. The "Investigation of suspected cancer cases" section consisted of three categories, namely, "proved cancer," "excluded cancer," and "strict follow-up because of disproved cancer."

\section{Definition of terms}

In this article, the term "FDG-PET" is defined as an examination performed with a PET scanner or PET/CT scanner. "FDG-PET cancer screening program" is defined as a cancer screening program using FDG-PET that is aimed at the detection of cancer at an early stage. Any PET or PET/CT detector, FDG-PET method, combined screening test, and method of further examination were included in the present report. Any method of selecting subjects and of handling the associated expense burden was allowed. Cases with a past history of cancer, or screening for recurrence requested by the attending physician or patient were excluded from the present report. However, when the cancer was considered to have been cured and FDG-PET was performed to screen for other sites of cancer, the FDG-PET test was included. "Combined screening tests," defined by each PET center, were those undergone by more than half of the subjects who underwent FDG-PET cancer screening at each PET center. If a screening test was performed at another facility within a short interval and the results were provided to the PET center, it was included in category of "combined screening tests." If further examination was judged necessary on the basis of the obtained PET information, any additional test other than the prefixed screening program was not considered to be a "combined screening test." If a cancer screening test was performed using a PET/CT scanner, regardless of the method used or manner of interpretation, the information obtained from the CT integrated in the PET/CT scanner was not regarded as a combined screening test, but as PET/ CT itself. This was because the CT findings could not be ignored when using PET/CT. Hence "PET positive" is defined as positive findings on PET or PET/CT. Accordingly, the cases with PET/negative and CT/positive findings on screening tests performed by PET/CT were defined as "PET/positive".

"Required further examination" is defined as cases for which a thorough examination was recommended on the basis of the comprehensive results of FDG-PET and/or any combined screening tests. Cases for which reexamination was recommended are not included in "required further examination." "Obtained result of further examination" is defined as being clearly categorized as "proved cancer," "excluded cancer," or "strict follow-up because of disproved cancer" through the results of additional examinations and treatment.

\section{Statistical analysis}

The Chi-square test for independence was performed to compare the detection rate, sensitivity, and positive predictive value between PET and PET-CT. A $P$ value of less than 0.05 was considered to be statistically significant.

\section{Results}

Characteristics of subjects who underwent FDG-PET cancer screening

The number of subjects in each age group is listed in Table 2. Subjects in their 50s and 60s made up the majority, accounting for $63.8 \%$ of all subjects. The rate of repeated annual FDG-PET cancer screening was $26.4 \%$ for all subjects.

A PET/CT scanner was used in 95,046 cases $(61.1 \%)$, and a dedicated PET scanner in 60,410 (38.9\%). In 2006,

Table 2 Number of subjects who underwent FDG-PET cancer screening program

\begin{tabular}{lrcrcrc}
\hline $\begin{array}{l}\text { Age } \\
\text { group }\end{array}$ & Number & $\begin{array}{l}\text { Rate } \\
(\%)\end{array}$ & Male & $\begin{array}{l}\text { Rate } \\
(\%)\end{array}$ & Female & $\begin{array}{l}\text { Rate } \\
(\%)\end{array}$ \\
\hline $10-19$ & 29 & 0.02 & 10 & 0.01 & 19 & 0.03 \\
$20-29$ & 1,008 & 0.7 & 474 & 0.5 & 534 & 0.9 \\
$30-39$ & 8,923 & 5.7 & 5,073 & 5.5 & 3,850 & 6.2 \\
$40-49$ & 26,206 & 16.9 & 15,764 & 17.0 & 10,442 & 16.8 \\
$50-59$ & 51,546 & 33.2 & 30,615 & 33.0 & 20,931 & 33.7 \\
$60-69$ & 47,712 & 30.7 & 29,382 & 31.7 & 18,330 & 29.5 \\
$70-79$ & 16,854 & 10.8 & 9,931 & 10.7 & 6,923 & 11.2 \\
$80-$ & 2,534 & 1.6 & 1,490 & 1.6 & 1,044 & 1.7 \\
Unknown & 644 & 0.4 & - & & - & \\
Total & 155,456 & & 92,739 & & 62,073 & \\
\hline
\end{tabular}


the number of different scanners used in the FDG-PET screening program was almost the same (dedicated PET scanner: 21,524 cases, PET/CT scanner: 21,527 cases). After 2007, the use of PET/CT scanners was higher than PET (in 2007: dedicated PET scanner: 15,217 cases, PET/ CT scanner: 24,650 cases; in 2008: dedicated PET scanner: 12,037 cases, PET/CT scanner: 26,892 cases, and in 2009: dedicated PET scanner: 11,629 cases, PET/CT scanner: 21,970 cases).

\section{FDG-PET method}

The FDG-PET scanning method was analyzed in 109 facilities with 171 scanners consisting of 44 PET scanners and $127 \mathrm{PET} / \mathrm{CT}$ scanners. Injected radioactivity of FDG was consistent in 38 facilities (range 111-370 MBq; average $188.2 \mathrm{MBq}$ ), and variable in 71 facilities. The facilities with variable injected radioactivities of FDG used $3.61 \mathrm{MBq}$ per kilogram body weight on average (range 2.6-5.0 MBq/kg), with total radioactivity of $216.9 \mathrm{MBq}$ on average (range 156-300 MBq) if used in subjects $165 \mathrm{~cm}$ tall and weighing $60 \mathrm{~kg}$. Maximum injected radioactivity of FDG ranged widely from 210 to $518 \mathrm{MBq}$ (average $338.4 \mathrm{MBq}$ ). Attenuation correction was performed in 108 facilities. All the attenuation correction with PET scanners was performed by conventional transmission scanning, and all the attenuation correction of PET/CT cameras was performed by CT scanning.

The start time of the emission scan was most frequently $60 \mathrm{~min}$ after injection of FDG (average $60.1 \mathrm{~min}$ ). Total required scanning time (including the scanning time of $\mathrm{CT}$ and transmission scan) was $25.5 \mathrm{~min}$ on average. The scanned range was most frequently "parietal to knee" regardless of whether a PET scanner or PET/CT scanner was used. Delayed scanning was performed regularly at 28 facilities, if necessary at 67 , and never at 22 facilities ( 8 facilities had multiple answers, and the decision depended on the scanner type used). The start time of delayed scanning was frequently $120 \mathrm{~min}$ after injection of FDG (average $117.9 \mathrm{~min}$ ).

\section{Characteristics of CT scanners integrated in the} PET/CT systems

The majority of PET centers used variable "mAs" values rather than a consistent $\mathrm{mAs}$ value (the product of X-ray tube current and exposure time) fixed to CT scanners integrated in the PET/CT system. Although the displayed CT scanning parameters on the machines and their definitions differ according to the manufacturer, the average displayed tube current (mAs), pitch, and computed tomography dose index-volume (CTDIvol) (mGy) of the scanner in facilities that applied a consistent $\mathrm{mAs}$ value to
CT scanning were $95.2 \mathrm{mAs}, 1.27$, and $6.2 \mathrm{mGy}$, respectively, whereas in facilities applying variable $\mathrm{mAs}$ values, they were $114.2 \mathrm{mAs}$ (maximum $\mathrm{mAs}$ value), 1.47 and $6.3 \mathrm{mGy}$, respectively. Tube voltage $(\mathrm{kV})$ was most frequently 120 or $140 \mathrm{kV}$.

The CT image reconstruction interval was most frequently 3.75 or $5 \mathrm{~mm}$. The location of the hands at the time of PET/CT scanning was elevated and pulled down at equal frequencies, and $\mathrm{CT}$ images were most frequently obtained during resting breathing.

\section{Combined screening tests}

The combined screening tests in the various facilities are listed in Table 3. A total of $85.5 \%$ of all facilities performed one or more combined screening tests in addition to FDG-PET. The number and type of combined screening tests differed by facility and by individual subjects even in the same facility. CT, abdominal ultrasonography (US), pelvic magnetic resonance imaging (MRI), fecal occult blood test (FOBT), and several kinds of serum tumor marker tests were most frequently adopted as combined screening tests with PET examination. On the other hand, physically taxing screening methods for the GI tract and colon tended to be avoided.

Characteristics of subjects with positive findings suggesting possible cancer

The number, gender, and age of subjects with positive findings suggesting possible cancer are listed in Table 4. Positive findings were noted in 16,955 cases (9,505 men, 7,352 women, and 98 gender unidentified). The rate of suspected cancer was $10.9 \%$ (annual yearly range $10.3-11.6 \%$ ) in total, which increased with age. The rate of suspected cancer in women was high in subjects aged 30-59 years due to the increased number of suspected thyroid and breast cancers.

The rate of suspected cancer was $9.8 \%$ (range 9.3-10.8\%) for dedicated PET combined with other screening examinations, lower than the rate of $11.6 \%$ (range 10.7-12.0 \%), which was found for PET/CT combined with other screening examinations $(P<0.01)$.

Analysis of suspected cancer according to the interpretation by FDG-PET and/or combined screening examination

Of the 16,955 cases with suspected cancer, the interpretation in 16,272 cases depended on the PET and combined screening test. Both PET positive and combined screening test positive were $20.4 \%$, PET positive only was $32.1 \%$, and combined screening test positive only was $47.5 \%$. Both dedicated PET and combined screening test positive 
Table 3 Combined screening tests and rate $(\%)$ of PET centers performing each test

\begin{tabular}{lrlc}
\hline Modalities & Rate $(\%)$ & Modalities & Rate $(\%)$ \\
\hline Brain MRI & 39.1 & Cervical cytology & 16.6 \\
Otolaryngologic examination & 0.6 & Pelvic ultrasonography & 19.1 \\
Head and neck CT & 27.1 & Pelvic CT & 32.3 \\
Head and neck MRI & 1.8 & Pelvic MRI & 48.1 \\
Thyroid ultrasonography & 37.4 & Prostate ultrasonography & 26.6 \\
Inspection and palpation of breast & 8.1 & Prostate MRI & 44.2 \\
Breast ultrasonography & 27.9 & Prostate specific antigen (PSA) & 82.2 \\
Mammography & 17.5 & Carcinoembryonic antigen (CEA) & 76.6 \\
Sputum cytology & 10.6 & Cancer antigen 19-9 (CA19-9) & 68.1 \\
Chest X-ray & 11.7 & Cancer antigen 125 (CA125) & 32.6 \\
Chest CT & 57.5 & व-fetoproteins (AFP) & 60.5 \\
Gastric fluoroscopy & 2.6 & Pepsinogen & 41.0 \\
Gastric endoscopy & 14.4 & Squamous cell carcinoma antigen (SCC) & 47.9 \\
Helicobacter pylori infection test & 11.9 & Cytokeratin-19 fragment (CYFRA) & 22.8 \\
Abdominal ultrasonography & 57.4 & Pro-gastrin releasing peptide (Pro-GRP) & 15.8 \\
Abdominal CT & 37.1 & Cancer antigen 15-3 (CA15-3) & 2.5 \\
Abdominal MRI & 15.8 & Neuron specific enolase (NSE) & 4.6 \\
Fecal occult blood test (FOBT) & 71.7 & Thyroglobulin & 11.3 \\
Barium enema & 0.0 & PIVKA-II & 7.5 \\
Colonoscopy & 1.9 & Urinary test & 13.8 \\
\hline
\end{tabular}

Analysis of detected cancers

Table 4 Number of subjects who were positive by screening FDG-PET and/or one or more of the combined screening tests if any

\begin{tabular}{lrrrrrr}
\hline $\begin{array}{l}\text { Age } \\
\text { group }\end{array}$ & Number & $\begin{array}{l}\text { Rate } \\
(\%)\end{array}$ & Male & $\begin{array}{l}\text { Rate } \\
(\%)\end{array}$ & Female & $\begin{array}{l}\text { Rate } \\
(\%)\end{array}$ \\
\hline $10-19$ & 0 & 0.0 & 0 & 0.0 & 0 & 0.0 \\
$20-29$ & 55 & 5.5 & 13 & 2.7 & 42 & 7.9 \\
$30-39$ & 653 & 7.3 & 285 & 5.6 & 368 & 9.6 \\
$40-49$ & 2,194 & 8.4 & 1,027 & 6.5 & 1,167 & 11.2 \\
$50-59$ & 5,240 & 10.2 & 2,900 & 9.5 & 2,340 & 11.2 \\
$60-69$ & 5,876 & 12.3 & 3,592 & 12.2 & 2,284 & 12.5 \\
$70-79$ & 2,437 & 14.5 & 1,427 & 14.4 & 1,010 & 14.6 \\
$80-$ & 402 & 15.9 & 261 & 17.5 & 141 & 13.5 \\
Unknown & 98 & 15.2 & - & - & - & - \\
Total & 16,955 & 10.9 & 9,505 & 10.2 & 7,352 & 11.8 \\
\hline
\end{tabular}

were $23.1 \%$, dedicated PET positive only was $27.5 \%$ and combined screening test positive only was $49.4 \%$. Both $\mathrm{PET} / \mathrm{CT}$ positive and combined screening test positive were $18.9 \%$, PET/CT scanner positive only was $34.6 \%$, and combined screening test positive only was $46.5 \%$. As a result, the estimated rate of suspected cancer was $5.0 \%$ with dedicated PET, $6.2 \%$ with PET/CT, and $7.4 \%$ with the combined screening tests (Fig. 1). The FDG-PETpositive rate was slightly higher in screening tests performed using PET/CT scanners than PET scanners $(P<0.01)$, and the positive rate with combined screening tests was much higher than FDG-PET $(P<0.01)$.
Of the 16,955 cases with suspected cancer, final results were obtained from 7,197 cases (42.4\%). The number of detected cancer cases was 1,912 , which amounted to $1.23 \%$ of the total number of cases screened. The number, gender, and age of the subjects in whom cancer was detected are listed in Table 5, and the number and kind of found cancers are listed in Table 6. Of the 1,912 cases of detected cancer, 1,491 had positive findings on PET (including PET/CT) amounting to $0.96 \%$ of the total screening number. As a result, $78.0 \%$ of detected cancers had positive PET findings. Of the 1,491 cases, 492 were detected by screening tests performed with PET scanners, and 999 cancers by screening tests performed with PET/CT scanners. The detection rate of cancer was higher with PET/CT scanner at $1.05 \%(999 / 95,039)$ than with PET scanner at $0.81 \%(492 / 60,407)(P<0.01)$. Of the 999 cancers detected by PET/CT scanners, 278 cases were positive only by PET, 594 cases were positive by PET and CT integrated with PET/CT and 127 cases were positive only by $\mathrm{CT}$ integrated in PET/CT.

According to the results of further examinations, the true positive rate for subjects with suggested possible cancer (positive predictive value) was $32.3 \%(1,491 / 4,609)$ with PET, with no statistically significant difference noted between PET/CT at $32.6 \%(1,000 / 3,069)$ and PET at $31.9 \%(491 / 1,540)$. Of the 7,197 cases in which a final result was obtained, repeated annual FDG-PET cancer 


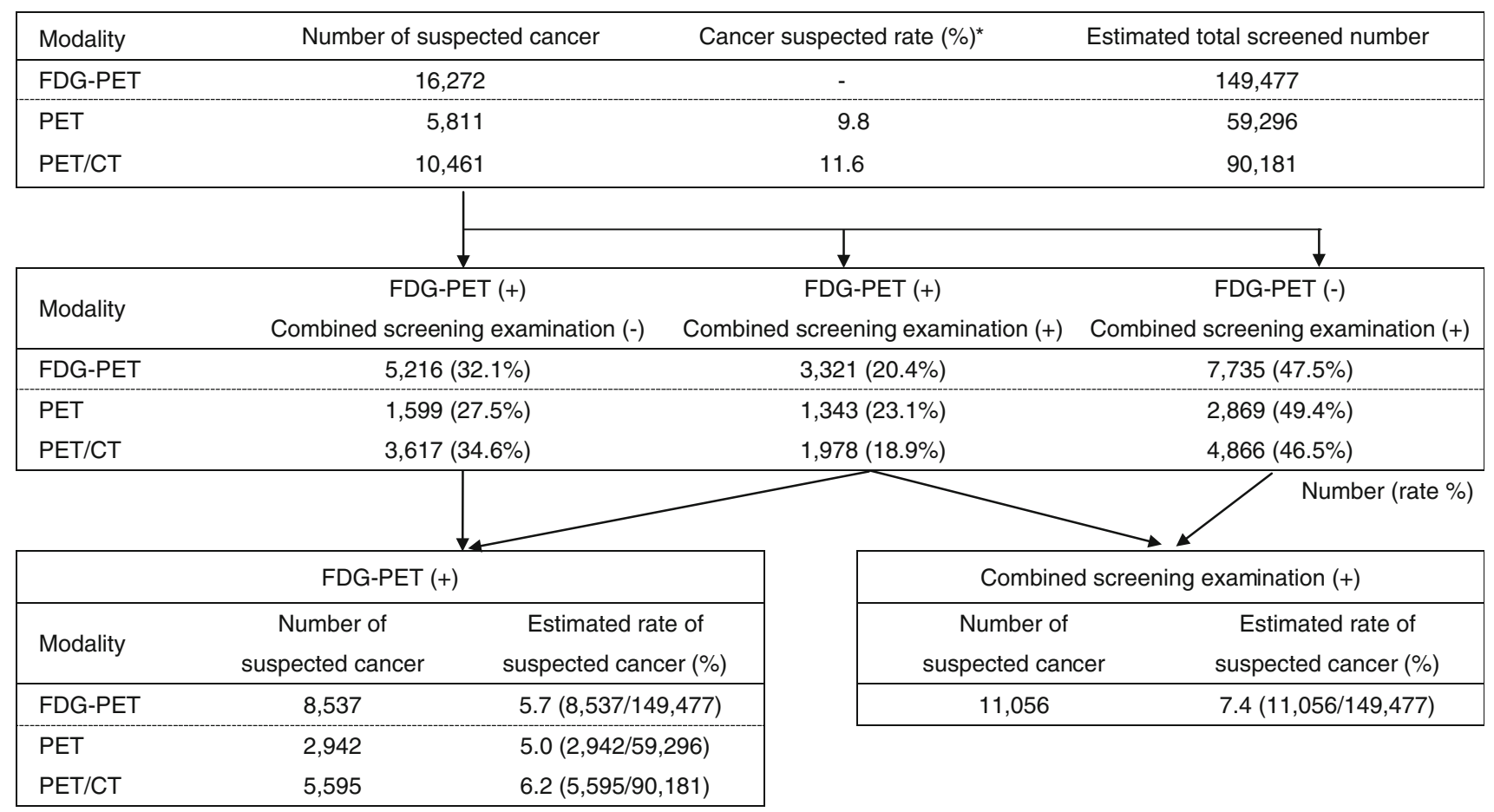

* Reference data obtained from this survey, (+): positive, (-): negative,

Fig. 1 Estimated rates of suspected cancer by FDG-PET and combined screening examination are shown. Of the 16,272 cases of suspected cancer by FDG-PET cancer screening program, 5,811 cases were suspected by dedicated PET and 10,461 cases by PET/CT. According to this survey, the cancer suspected rate was $9.8 \%$ for dedicated PET combined with other screening examinations and
$11.6 \%$ for PET/CT combined with other screening examinations. The estimated number receiving FDG-PET cancer screening program was 59,296 for dedicated PET combined with other screening examinations and 90,181 for PET/CT combined with other screening examinations. Finally, the total number of subjects who received FDG-PET cancer screening program was estimated to be 149,477
Table 5 Number and rate of found cancers which was positive by screening FDG-PET and/or one or more of the combined screening tests if any

\begin{tabular}{|c|c|c|c|c|c|c|c|c|c|}
\hline \multirow{2}{*}{$\begin{array}{l}\text { Age } \\
\text { group }\end{array}$} & \multicolumn{3}{|l|}{ Total } & \multicolumn{3}{|l|}{ Male } & \multicolumn{3}{|l|}{ Female } \\
\hline & Number & $\begin{array}{l}\text { Found } \\
\text { cancer }\end{array}$ & PPV (\%) & Number & $\begin{array}{l}\text { Found } \\
\text { cancer }\end{array}$ & PPV (\%) & Number & $\begin{array}{l}\text { Found } \\
\text { cancer }\end{array}$ & PPV (\%) \\
\hline $10-19$ & 0 & 0 & 0 & 0 & 0 & 0 & 0 & 0 & 0 \\
\hline $20-29$ & 14 & 0 & 0 & 3 & 0 & 0.0 & 11 & 0 & 0 \\
\hline $30-39$ & 218 & 36 & 16.5 & 83 & 12 & 14.5 & 135 & 24 & 17.8 \\
\hline $40-49$ & 868 & 199 & 22.9 & 355 & 71 & 20.0 & 513 & 128 & 25.0 \\
\hline $50-59$ & 2,282 & 549 & 24.1 & 1,196 & 282 & 23.6 & 1,086 & 267 & 24.6 \\
\hline $60-69$ & 2,593 & 711 & 27.4 & 1,553 & 468 & 30.1 & 1,040 & 243 & 23.4 \\
\hline $70-79$ & 1,063 & 352 & 33.1 & 632 & 230 & 36.4 & 431 & 122 & 28.3 \\
\hline 80- & 159 & 65 & 40.9 & 103 & 45 & 43.7 & 56 & 20 & 35.7 \\
\hline Total & 7,197 & 1,912 & 26.6 & 3,925 & 1,108 & 28.2 & 3,272 & 804 & 24.6 \\
\hline
\end{tabular}

$P P V$ positive predictive value

screening tests were performed in 1,240 cases, with cancer found in $22.9 \%$ of them, lower than that in the unrepeated cases $(27.5 \%)$.

The cancers found in the FDG-PET cancer screening program were of a wide variety, as listed in Table 6. The most frequently detected cancers with high PET-positive rates were colon/rectum cancer (396 cases, $85.9 \%$ ), thyroid cancer (353 cases, $90.7 \%$ ), lung cancer (319 cases,
$86.8 \%$ ), and breast cancer (163 cases, 84.0 \%). Low PETpositive rates, but high positive rates in combined screening tests were found with prostate cancer (165 cases, $37.0 \%$ ) and gastric cancer (124 cases, $37.9 \%)$. Renal cancer, malignant lymphoma, pancreas cancer, esophagus cancer, uterine cancer, and bladder cancer were also frequently found. Cancers were most frequently found in the age groups of 50-59 years and 60-69 years, accounting for 
Table 6 Number of cancers detected by the screening program

\begin{tabular}{llll}
\hline Disease & Number & Disease & Number \\
\hline Colorectal cancer & 396 & Thymic tumor & 8 \\
Thyroid cancer & 353 & Bile duct cancer & 6 \\
Lung cancer & 319 & Gallbladder cancer & 5 \\
Prostate cancer & 165 & Sarcoma & 5 \\
Breast cancer & 163 & Testicular cancer & 4 \\
Gastric cancer & 124 & Small intense tumor & 4 \\
Renal cancer & 59 & Primary unknown cancer & 4 \\
Malignant lymphoma & 53 & Gastrointestinal stromal tumor (GIST) & 3 \\
Pancreas cancer & 45 & Brain tumor & 3 \\
Esophagus cancer & 38 & Metastatic bone tumor & 3 \\
Uterine cancer & 35 & Pleural tumor & 2 \\
Bladder cancer & 30 & Peritoneal cancer & 1 \\
Liver cancer & 27 & Adrenal cancer & 1 \\
Head and neck cancer & 22 & Urethra cancer & 1 \\
Ovarian cancer & 21 & Urachal tumor & 1 \\
Bone marrow disease & 10 & Skin cancer & 1 \\
& & Total & 1,912 \\
\hline
\end{tabular}

Table 7 PET-positive rate and number of found cancer according to UICC Stage

\begin{tabular}{|c|c|c|c|c|c|c|c|}
\hline & \multirow[t]{2}{*}{ UICC Stage } & \multicolumn{6}{|l|}{ Kind of cancer } \\
\hline & & Lung & Colon & Breast & Thyroid & Prostate & Stomach \\
\hline \multirow[t]{5}{*}{ FDG-PET screening program } & 0 & - & $76.7(33 / 43)$ & $81.9(9 / 11)$ & - & - & - \\
\hline & I & $84.2(117 / 139)$ & $85.3(93 / 109)$ & $82.1(55 / 67)$ & $99.0(95 / 96)$ & $25.0(8 / 32)$ & $8.8(3 / 31)$ \\
\hline & II & $100.0(6 / 6)$ & $77.8(7 / 9)$ & $91.6(11 / 12)$ & $100.0(9 / 9)$ & $33.3(2 / 6)$ & $75.0(3 / 4)$ \\
\hline & III & $100.0(26 / 26)$ & $100.0(21 / 21)$ & $100.0(1 / 1)$ & $90.0(9 / 10)$ & $25.0(2 / 8)$ & $100.0(5 / 5)$ \\
\hline & IV & $100.0(23 / 23)$ & $100.0(20 / 20)$ & $100.0(5 / 5)$ & $100.0(7 / 7)$ & $80.0(4 / 5)$ & $100.0(7 / 7)$ \\
\hline \multirow[t]{5}{*}{ Repeated FDG-PET cancer program } & 0 & - & $50.0(1 / 2)$ & $50.0(1 / 2)$ & - & - & - \\
\hline & I & $80.6(25 / 31)$ & $88.9(8 / 9)$ & $57.1(8 / 14)$ & $100.0(4 / 4)$ & $33.3(2 / 6)$ & $22.2(2 / 9)$ \\
\hline & II & - & - & - & $100.0(1 / 1)$ & - & - \\
\hline & III & $100.0(1 / 1)$ & $100.0(2 / 2)$ & $100.0(1 / 1)$ & - & - & - \\
\hline & IV & $100.0(2 / 2)$ & $100.0(1 / 1)$ & - & $100.0(1 / 1)$ & $100.0(2 / 2)$ & - \\
\hline
\end{tabular}

Numbers in parentheses represent number of PET-positive case/number of total screened cancer

UICC The Union for International Cancer Control

$66.9 \%$ of the total found cancers. Cancers were most frequently found in the age group of 60-69 years in men and $50-59$ years in women.

\section{Clinical stage for six representative kinds of cancers}

Table 7 summarizes the result of PET according to Union for International Cancer Control (UICC) clinical stage for six representative kinds of cancers (lung, colon/rectum, thyroid, breast, prostate and stomach). Cancer of the lung, colon/rectum, thyroid and breast was found mostly at Stage I with high PET sensitivity. Most prostate and gastric cancers were also categorized as Stage I, but they were largely found with the combined screening tests. The cancers found with repeated FDG-PET cancer screening were commonly found at Stage I, but a small number of cancers were found in advanced stages.

Analysis of detected non-cancerous lesions

According to the results of further examinations for subject with positive findings in FDG-PET screening program, many non-cancerous lesions were found. The major non-cancerous lesions found are listed in Table 8. Most of the non-cancerous lesions were found with PET or PET/CT. However, FDG-PET or PET/CT had moderate to low sensitivity for colonic diverticulum (45.7\%), benign prostatic hypertrophy $(35.1 \%)$, liver hemangioma 
Table 8 Number of benign lesions detected by the screening program

\begin{tabular}{llll}
\hline Disease & Number & Disease & Number \\
\hline Colonic adenoma (polyp) & 687 & Gastric polyp & 41 \\
Nodular goiter & 457 & Uterine myoma & 40 \\
Benign prostate hypertrophy & 114 & Benign parotid tumor & 30 \\
Old inflammatory change of lung & 113 & Mammary cyst & 29 \\
Inflammatory change of lung & 99 & Liver hemangioma & 28 \\
Colonic diverticulum & 92 & Thyroid cyst & 27 \\
Chronic thyroiditis & 90 & Ovarian cyst & 19 \\
Colitis & 66 & Neurogenic tumor & 19 \\
Mastopathy & 65 & Endometriosis & 14 \\
Hemorrhoid & 64 & Sarcoidosis & 14 \\
Follicular goiter & 46 & Tuberculosis & 10 \\
Mammary fibroadenoma & 45 & Adenoid & 10 \\
\hline
\end{tabular}

(28.6\%), mammary cyst $(27.6 \%)$ and gastric polyp $(7.3 \%)$, which tended to be found with the combined screening tests.

\section{Discussion}

There have been many arguments regarding the application of FDG-PET for cancer screening [1, 4-6]. PET examination has the potential to detect many types of malignant neoplasms; therefore, cancer screening with PET is expected to detect malignant neoplasms throughout the body. However, it is well known that PET has limited detectability for several types of malignant neoplasms due to their small size, low glucose metabolism, and high physiological background FDG uptake. As a result, researchers involved in cancer screening using FDG-PET share a common understanding that cancer screening using PET or PET/CT must be established as a program in combination with several other modalities to compensate for what is not apparent with PET and PET/CT [2, 3, 5, 6]. The Japanese Society of Nuclear Medicine published guidelines for "FDG-PET cancer screening", which include details about common examinations adopted in Japan that have the potential to contribute to an increased cancer detection rate. These guidelines facilitate uniform and accurate PET cancer screening at many PET centers, but do not advocate any particular screening methods to be combined with PET examination. Therefore, PET centers modify their cancer screening programs depending on their circumstances (even though they share some common ground, as shown in the present report). The suspected cancer detection rate with combined screening tests is much higher than that of FDG-PET; thus, adoption of a common combined screening test and improvement in accuracy will contribute to increasing the specificity of the FDG-PET cancer screening program. The rate of suspected cancer was higher in PET/CT combined with other screening examinations than dedicated PET combined with other screening examinations. From the result of suspected cancer according to the interpretation of FDG-PET and/or combined screening examinations, the estimated rate of suspected cancer was higher with PET/CT than PET. As a result, PET/CT increased the rate of suspected cancer, but this was not associated with any specific type of cancer.

The age-specific incidence of cancer shows a sudden rise after 60 years of age in Japan based on the report from the Foundation for Promotion of Cancer Research (FPCR) [7]. The number of FDG-PET cancer screening programs is highest for subjects aged 50-59 years $(33.2 \%$ of all subjects), and the incidence of cancer increases with age. Subjects receiving FDG-PET cancer screening are younger than the reported age groups with the most frequent incidence of cancer. The number of malignant neoplasms found by the FDG-PET cancer screening program is higher than the age-matched annual incidence of cancer in Japan. The distinctive feature of FDG-PET cancer screening is that it can detect various kinds of cancers, including cancers with unestablished organized screening methodologies. Moreover, the majority of found cancers were of Stage I, with expected good prognoses with existing treatment methods. The incidence of cancer in this survey is similar to those in several reports from single or multicenter facilities [3, 5, 8-13], indicating that PET cancer screening is conducted with a considerable level of consistency throughout Japan. Detection rates with FDG-PET cancer screening are higher than the total detection rates of other cancer screening modalities performed in Japan (such as chest radiography and sputum cytology, mammography with inspection and palpation of breast, upper gastrointestinal fluoroscopy, FOBT, and uterine cervical cytology), which have already proven cancer screening efficacy [14]. Terauchi et al. [14] reported strict limitations of FDG-PET for cancer screening based on data from the Research 
Center for Cancer Prevention and Screening (RCCPS) and National Cancer Center in Japan, which were particularly notable in screening for colon cancer, lung cancer and gastric cancer. All found lung cancers were categorized as Stage I, and most found colon cancers were localized in the intramucosal layer. The results of the present report indicate that cancer screening by PET examination alone was limited to small and/or early cancers; therefore, combined examinations to detect cancers missed by FDG-PET is mandatory. On the other hand, combined examinations are associated with an increased number of false positives, and can lead to an increase in unnecessary further examinations and follow-up. Combined screening tests must be specific for a particular cancer. FDG-PET cancer screening is not a completely established method for cancer screening. FDGPET cancer screening should be used with a sufficient explanation and adequate understanding of its advantages, disadvantages and limitations.

One problem is the appropriate time interval for conducting FDG-PET cancer screening. The present results show that cancer was found in $22.9 \%$ of suspected cancer cases in which repeated annual FDG-PET cancer screening tests were performed. Although most cancers found in the repeated annual FDG-PET cancer screening tests were Stage I, the cancer detection rate was not low. Estimated from the data of the HIMEDIC Imaging Center at Lake Yamanaka, cancer was found in $1.58 \%$ of cases at the first screening, and in $1.18 \%$ of cases at the second screening, but only in $0.69 \%$ of cases in the third screening. The detection rate was estimated to decrease in PET examinations, but was stable in the combined screening test at a rate of approximately $0.2 \%$ [2]. The present results showed that cancer at Stage I was frequently found by FDG-PET cancer screening, with a high sensitivity of FDG-PET for both the first and repeated examinations. These results highlight the limitation of a one-time FDG-PET cancer screening program, and that two consecutive screenings followed by a combined screening test may be able to detect more malignancies. Among subjects who underwent a repeated FDG-PET cancer screening program, Stage III and IV cancers comprised $11.4 \%$ of the found cancers. Found cancers in a repeated FDG-PET screening program may be interpreted as negative on the first time examination or show a rapid onset within 1 year. It is absolutely clear that the FDG-PET cancer screening program cannot detect all malignancies.

The main issue for FDG-PET cancer screening will be how it can detect various cancers and reduce their mortality rate. FDG-PET cancer screening detected many prostate cancers and thyroid cancers, which are associated with relatively long survival times. In contrast, FDG-PET cancer screening programs detected pancreas cancer at an early clinical stage [15], but the high mortality rate of even early-stage pancreas cancer means that the FDG-PET cancer screening program may be non-contributory for this tumor. Although a randomized control study is a maximally efficient method for showing the benefit of FDGPET cancer screening, it requires a long observation time and a large population. The United States Preventive Services Task Force (USPSTF) advocated an "analytic framework" consisting of chain logic of evidence supporting a connection between the preventive service and improved health outcomes [16]. As a result, the value of FDG-PET cancer screening might be indirectly estimated by detailed analysis of found cancers compared to the known epidemiological trend of cancer. Moreover, decision modeling analysis may also have the potential to clarify the clinical benefit of a PET cancer screening program [17], even though direct evidence such as reduction of mortality is essential.

Radiation exposure is a major problem in FDG-PET cancer screening. According to the International Commission on Radiation Protection (ICRP), the estimated cancer incidence induced by radiation exposure was $0.0048 \%$ per $\mathrm{mSv}$ [13]. Murano et al. reported that the average effective dose of radiation exposure in a PET center is estimated to be $4.4 \mathrm{mSv}$ with a dedicated PET study and $14.2 \mathrm{mSv}$ with a PET/CT study. The average radiation exposure associated with FDG-PET cancer screening will increase because of the increasing availability of PET/CT scanners. Riskbenefit analysis has shown that FDG-PET cancer screening is beneficial for examinees above the break-even ages based on a Japanese nationwide survey. However, that survey mentioned that the risks and benefits of radiation exposure must be explained to examinees because of the larger radiation dose used in cancer FDG-PET screening compared with other radiological tests [18].

The high cost of an FDG-PET cancer screening program is another huge problem. Effective combined screening tests, at the minimum, for compensating for FDG-PET should be selected as a screening program, which can lead to a lower cost and burden on the recipients of such screening. Although the cost of FDG-PET in Japan is lower than that in the United States $(\$ 1,000$ vs. $\$ 2,000$, respectively) [1, 19], it is still far higher than that of a screening examination covered by health insurance plans. Therefore, cost effectiveness is not expected in an FDG-PET cancer screening program. If maximum cost effectiveness is required for an FDG-PET cancer screening program, the program should target a group at high risk for a cancer that is commonly detected by PET and PET/CT. Otherwise, the value of a FDG-PET cancer screening program is left to the judgment of the individual provided that the advantages, disadvantages and limitations of FDG-PET for cancer screening are fully disclosed.

Beside malignancies, many benign lesions were found with the FDG-PET cancer screening program. They are 
regarded as false positive cases. However, colonic adenoma has the potential for malignant transformation $[20,21]$, and screening and removal of adenomatous polyps are significant aspects of prevention and improvement of prognosis. The clinical importance of screening for adenomas is to detect advanced adenomas (defined as larger adenomas with a diameter of $10 \mathrm{~mm}$ or more). The sensitivity of FDG-PET for detecting adenomas depends on their size and histologic grade [22, 23]. Thus, FDG-PET has a high potential of detecting adenomas that should be removed and suggesting the need for further examinations such as colonoscopy. Moreover, some non-malignant diseases (such as tuberculosis, inflammatory changes of the lung, and sarcoidosis) are also clinically important, and in some instances, early detection may enhance the quality of life and longevity. Therefore, FDG-PET screening may provide an opportunity to favorably alter the prognosis in various cases.

A limitation of this survey is inadequate investigation of subjects who were judged to be negative in the FDG-PET cancer screening program. Also, the present survey received inadequate answers from subjects who were judged as having possible malignancy by the FDG-PET cancer screening program, even though proven malignancies appeared to be obtained more frequently.

\section{Conclusion}

The FDG-PET screening program in Japan has detected a variety of cancers at an early stage. However, several cancers were found in repeated FDG-PET cancer screening program, indicating an important limitation of a one-time FDG-PET cancer screening program. The value of the FDG-PET cancer screening program is left to the judgment of individuals with regard to its potentials and limitations.

\begin{abstract}
Acknowledgments This work was supported by the National Cancer Center Research and Development Fund 23-A-25, Japanese Council of PET Imaging: working group on epidemiology study for PET cancer screening, the PET cancer screening research group of "Yokohama City University". The authors would like to express our sincere appreciation and gratitude for Sachiko Yanagida from Japan Radioisotope Association for management of this survey and data, and Yoshihide Nakamura from Japan Radioisotope Association for management of this survey.
\end{abstract}

Conflict of interest The authors declare no conflict of interest.

\section{References}

1. Schöder H, Gönen M. Screening for cancer with PET and PET/ CT: potential and limitations. J Nucl Med. 2007;48(Suppl 1): 4S-18S

2. Ide M. Cancer screening with FDG-PET. Q J Nucl Med Mol Imaging. 2006;50:23-7.
3. Minamimoto R, Senda M, Uno K, Jinnouchi S, Iinuma T, Ito K, et al. Performance profile of FDG-PET and PET/CT for cancer screening on the basis of a Japanese Nationwide Survey. Ann Nucl Med. 2007;21:481-98.

4. Weckesser M, Schober O. Is whole-body FDG-PET valuable for health screening? Against. Eur J Nucl Med Mol Imaging. 2005; 32:342-3.

5. Ide M, Suzuki Y. Is whole-body FDG-PET valuable for health screening? For. Eur J Nucl Med Mol Imaging. 2005;32:339-41.

6. Yasuda S, Ide M, Fujii H, Nakahara T, Mochizuki Y, Takahashi $\mathrm{W}$, et al. Application of positron emission tomography imaging to cancer screening. Br J Cancer. 2000;83:1607-11.

7. The Foundation for Promotion of Cancer Research (FPCR) Cancer Statistics in Japan 2010 http://ganjoho.jp/public/statistics/ backnumber/2010_en.html.

8. Ono K, Ochiai R, Yoshida T, Kitagawa M, Omagari J, Kobayashi $\mathrm{H}$, et al. The detection rates and tumor clinical/pathological stages of whole-body FDG-PET cancer screening. Ann Nucl Med. 2007;21:65-72.

9. Kojima S, Zhou B, Teramukai S, Hara A, Kosaka N, Matsuo Y, et al. Cancer screening of healthy volunteers using whole-body F-18 FDG-PET scans: the Nishidai clinic study. Eur J Cancer. 2007;43:1842-8.

10. Nishizawa S, Kojima S, Teramukai S, Inubushi M, Kodama H, Maeda Y, et al. Prospective evaluation of whole-body cancer screening with multiple modalities including [18F]fluorodeoxyglucose positron emission tomography in a healthy population: a preliminary report. J Clin Oncol. 2009;27:1767-73.

11. Chen YK, Ding HJ, Su CT, Shen YY, Chen LK, Liao AC, et al. Application of PET and PET/CT imaging for cancer screening. Anticancer Res. 2004;24:4103-8.

12. Ko DH, Choi JY, Song Y, Lee SJ, Kim YH, Lee K, et al. The usefulness of 18F-FDG PET as a cancer screening test. Nucl Med Mol Imaging. 2008;42:444-50.

13. Lee JW, Kang KW, Paeng JC, Lee SM, Jang SJ, Chung JK, et al. Cancer screening using 18F-FDG PET/CT in Korean asymptomatic volunteers: a preliminary report. Ann Nucl Med. 2009; 23:685-91.

14. Terauchi T, Murano T, Daisaki H, Kanou D, Shoda H, Kakinuma $\mathrm{R}$, et al. Evaluation of whole-body cancer screening using $18 \mathrm{~F}-$ 2-deoxy-2-fluoro-D-glucose positron emission tomography: a preliminary report. Ann Nucl Med. 2008;22:379-85.

15. Minamimoto R, Senda $M$, Terauchi $T$, Jinnouchi S, Inoue $T$, Iinuma $\mathrm{T}$, et al. Analysis of various malignant neoplasms detected by FDG-PET cancer screening program: based on a Japanese Nationwide Survey. Ann Nucl Med. 2011;25:45-54.

16. Harris RP, Helfand M, Woolf SH, Lohr KN, Mulrow CD, Teutch SM, et al. Current methods of the US Preventive Services Task Force: a review of the process. Am J Prev Med. 2001;20:21-35.

17. Vach W, Flemming P, Hoilund-Carlsen PF, Gerke O, et al. Generating evidence for clinical benefit of PET/CT in diagnosing cancer patients. J Nucl Med. 2011;52:77S-85S.

18. Murano T, Minamimoto R, Senda M, Uno K, Jinnouchi S, Fukuda $\mathrm{H}$, et al. Radiation exposure and risk-benefit analysis in cancer screening using FDG-PET: results of a Japanese nationwide survey. Ann Nucl Med. 2011;25:657-66.

19. Yasunaga H. Who wants cancer screening with PET? A contingent valuation survey in Japan. Eur J Radiol. 2009;70:190-4.

20. Bond JH. Polyp guideline: diagnosis, treatment, and surveillance for patients with colorectal polyps. Practice Parameters Committee of the American College of Gastroenterology. Am J Gastroenterol. 2000;95:3053-63.

21. Chen CD, Yen MF, Wang WM, Wong JM, Chen TH. A casecohort study for the disease natural history of adenomacarcinoma and de novo carcinoma and surveillance of colon and rectum after 
polypectomy: implication for efficacy of colonoscopy. $\mathrm{Br} \mathrm{J}$ Cancer. 2003;88:1866-73.

22. Prabhakar HB, Sahani DV, Fischman AJ, Mueller PR, Blake MA. Bowel hot spots at PET-CT. Radiographics. 2007;27:145-59.
23. Nakajo M, Jinnouchi S, Tashiro Y, Shirahama H, Sato E, Koriyama C, et al. Effect of clinicopathologic factors on visibility of colorectal polyps with FDG PET. AJR Am J Roentgenol. 2009;192:754-60. 